\title{
MULTI-WAVELENGTH EMISSION FROM THE FERMI BUBBLE. II. SECONDARY ELECTRONS AND THE HADRONIC MODEL OF THE BUBBLE
}

\author{
K.-S. Cheng ${ }^{1}$, D. O. Chernyshov ${ }^{1,2}$, V. A. Dogiel ${ }^{1,2,4}$, And C.-M. Ko ${ }^{3}$ \\ ${ }^{1}$ Department of Physics, University of Hong Kong, Pokfulam Road, Hong Kong, China \\ ${ }^{2}$ I. E. Tamm Theoretical Physics Division of P. N. Lebedev Institute of Physics, Leninskii pr. 53, 119991 Moscow, Russia \\ ${ }^{3}$ Institute of Astronomy, Department of Physics and Center for Complex Systems, National Central University, Jhongli, Taiwan \\ ${ }^{4}$ Moscow Institute of Physics and Technology (State University), 9, Institutsky Lane, Dolgoprudny 141707, Russia \\ Received 2014 May 31; accepted 2014 November 21; published 2015 January 20
}

\begin{abstract}
We analyze the origin of the gamma-ray flux from the Fermi Bubbles (FBs) in the framework of the hadronic model in which gamma-rays are produced by collisions of relativistic protons with the protons of the background plasma in the Galactic halo. It is assumed in this model that the observed radio emission from the FBs is due to synchrotron radiation of secondary electrons produced by $p p$ collisions. However, if these electrons lose their energy through synchrotron and inverse-Compton emission, the spectrum of secondary electrons will be too soft, and an additional arbitrary component of the primary electrons will be necessary in order to reproduce the radio data. Thus, a mixture of the hadronic and leptonic models is required for the observed radio flux. It was shown that if the spectrum of primary electrons is $\alpha E_{e}^{-2}$, the permitted range of the magnetic field strength is within the 2-7 $\mu \mathrm{G}$ region. The fraction of gamma-rays produced by $p p$ collisions can reach about $80 \%$ of the total gamma-ray flux from the FBs. If the magnetic field is $<2 \mu \mathrm{G}$ or $>7 \mu \mathrm{G}$ the model is unable to reproduce the data. Alternatively, the electrons in the FBs may lose their energy through adiabatic energy losses if there is a strong plasma outflow in the GC. Then, the pure hadronic model is able to reproduce characteristics of the radio and gamma-ray flux from the FBs. However, in this case the required magnetic field strength in the FBs and the power of CR sources are much higher than those following from observations.
\end{abstract}

Key words: acceleration of particles - Galaxy: center - gamma rays: ISM

\section{INTRODUCTION}

The origin of the giant gamma-ray structure called Fermi bubbles (FBs), discovered from analysis of the Fermi Large Area Telescope data (see Dobler et al. 2010; Su et al. 2010; Ackermann et al. 2014), is still enigmatic. Two main interpretations of the gamma-ray production in the bubbles have been suggested, which can be defined as hadronic and leptonic. In the latter case gamma-rays are produced by inverse-Compton scattering of relativistic electrons off background photons (see, e.g., Su et al. 2010). Because of the relatively short lifetime of relativistic electrons, they should be produced inside or near regions of emission. The in situ acceleration can be provided either by a shock or shocks which are generated by tidal processes near the central black hole or by an MHD turbulence that is excited behind a shock (for some aspects of the leptonic model, see, e.g., Cheng et al. 2014; Mertsch \& Sarkar 2011; Yang et al. 2012, and others).

Alternatively, the hadronic model of the FBs was suggested and developed in a series of publications by Crocker \& Aharonian (2011), Crocker et al. (2011, 2013), Fujita et al. (2013), Thoudam (2013), and Yang et al. (2014). This model does not require in situ acceleration of protons because of their relatively long lifetime. Thus, Crocker \& Aharonian (2011) concluded from observational data that star formation regions within the central $200 \mathrm{pc}$ radius release energy continuously, with the average power of $\sim 10^{40} \mathrm{erg} \mathrm{s}^{-1}$. They assumed that this energy is transformed into a flux of relativistic cosmic rays (CRs) which are carried away into the halo by plasma outflow from the Galactic Center (GC). They also assumed that CR protons fill the bubble region and are somehow trapped there for the duration of $p p$ collisions, $\tau_{p p} \sim\left(n_{H} \sigma_{p p} c\right)^{-1} \sim 10^{10} \mathrm{yr}$, where $\sigma_{p p}$ is the cross-section of $p p$ collisions and $n_{H} \simeq 10^{-2} \mathrm{~cm}^{-3}$ is the plasma density in the halo.
According to Yang et al. (2014), the leptonic model is unable to interpret the observed hardening of the gamma-ray spectrum in the FB edges, which is a problem in the leptonic model. We suppose, however, that special investigations of this effect are necessary in order to get a more reliable conclusion.

The hadronic scenario requires a rather small diffusion coefficient of protons within the bubble, $\sim 10^{26} \mathrm{~cm}^{2} \mathrm{~s}^{-1}$ at $1 \mathrm{GeV}$ (see Crocker \& Aharonian 2011), which is two orders of magnitude smaller than in the Galaxy, or some sort of "magnetic wall" near the FB edges is needed to confine protons in the FBs for a long time (see Jones et al. 2012).

In these assumptions, a power in the $\mathrm{CR}$ proton of about $W \simeq 2 \times 10^{38} \mathrm{erg} \mathrm{s}^{-1}$ is required in order to produce the gamma-ray flux $F_{\gamma} \simeq 4 \times 10^{37} \mathrm{erg} \mathrm{s}^{-1}$ from the FBs. Such a power can easily be supplied by star formation regions in the GC. The spectrum of gamma-rays from the FBs can be presented as a power law,

$$
F_{\gamma}^{\mathrm{FB}} \propto E_{\gamma}^{-2}
$$

in the range $E_{\gamma}=1-100 \mathrm{GeV}$ (see Su et al. 2010). Crocker \& Aharonian (2011) estimated a radio flux from FBs produced by secondary electrons generated by $p p$ collisions. They obtained that the radio flux in the range $20-60 \mathrm{GHz}$ is about $\Phi_{v} \simeq$ $2 \times 10^{36} \mathrm{erg} \mathrm{s}^{-1}$ for the magnetic field strength $H>10 \mu \mathrm{G}$; this is about the same as the flux derived from Finkbeiner (2004) from the WMAP data, $\Phi_{v} \simeq(1-5) \times 10^{36} \mathrm{erg} \mathrm{s}^{-1}$. These and subsequent observations of radio emission from the FBs (see Jones et al. 2012; Ade et al. 2013) showed that the FB radio spectrum is a power law:

$$
\Phi_{v}^{\mathrm{FB}} \propto v^{-\alpha},
$$

where $\alpha$ is the spectral index of the radio emission, which ranges from 0.5 to 0.63 at $\mathrm{GHz}$ frequencies. If the emission is synchrotron emission, then the spectrum of the radio emitting 
electrons is close to a power law,

$$
N_{e} \propto E_{e}^{-(2 \alpha+1)} .
$$

Below we would like to derive the parameters of the hadronic model, where the main part of the radio and gamma-ray emission from the FB can be produced, indeed, by proton interaction with the gas in the Galactic Halo (hadronic model).

\section{SPECTRA OF GAMMA-RAYS AND SECONDARY ELECTRONS IN THE HADRONIC MODEL}

In the hadronic model, when protons are trapped in the FBs and lose energy via $p p$ collisions, their spectrum, $N_{p}(E)$, can be described in the framework of the "leaky box model" (for details, see Berezinskii et al. 1990)

$$
n_{H} \sigma_{p p} c N_{p}(E)=Q(E)
$$

where the constant $Q(E)$ is the power of the proton sources in the GC.

The intensity of the $p p$ gamma-ray emission can be calculated from

$$
I_{p p}\left(E_{\gamma}\right)=\frac{n_{H} c L}{4 \pi} \int \frac{d \sigma_{p p}\left(E, E_{\gamma}\right)}{d E_{\gamma}} N_{p}(E) d E,
$$

where $d \sigma_{p p} / d E_{\gamma}$ is the differential cross-section of the gammarays produced (see, e.g., Kamae et al. 2006; Shibata et al. 2013), and $L$ is the thickness of the radiating region. Below we calculate intensities of gamma-ray and microwave emission at relatively high latitudes where the densities of the gas, photons, magnetic field strength, and CR protons are supposed to vary relatively slowly along the path of view. Therefore, we neglect their spatial variations in the halo and use average values of these components there.

If the FB gamma-ray flux is generated by $p p$ collisions of relativistic protons with protons of the background plasma, then as follows from Equation (1) the spectrum of the protons needed to reproduce the flux of gamma-rays from the FBs is (see Crocker \& Aharonian 2011)

$$
N_{p}(E)=K_{p} E^{-2} \theta\left(E_{\max }^{p}-E\right),
$$

where $K_{p}$ is a constant and $E_{\max }^{p}$ is the maximum energy of the emitting protons whose value can be estimated from the cut-off position, $E_{\gamma}^{\text {cut-off }}$, in the FB gamma-ray spectrum. According to the recent data analysis of Ackermann et al. (2014), the cut-off position is $E_{\gamma}^{\text {cut-off }} \leqslant 200 \mathrm{GeV}$, which gives the estimate for $E_{\max }^{p} \simeq 3 \mathrm{TeV}$ (see Atoyan 1992).

The production spectrum of secondary electrons is described by the equation

$$
Q\left(E_{e}\right)=n_{H} c \int \frac{d \sigma\left(E, E_{e}\right)}{d E_{e}} N_{p}(E) d E,
$$

where $d \sigma / d E_{e}$ is the differential cross-section of the secondary electrons produced by $p p$ collisions (see, e.g., Kamae et al. 2006; Shibata et al. 2013).

In the framework of the "leaky box model" the spectrum of secondary electrons in the FBs is

$$
N_{s e}\left(E_{e}\right)=\frac{1}{|d E / d t|} \int_{E_{e}}^{\infty} Q\left(E_{e}^{\prime}\right) d E_{e}^{\prime}
$$

where $d E / d t$ is the rate of electron energy losses. Relativistic electrons in the halo lose their energy by synchrotron and inverse-Compton emission (see Blumenthal \& Gould 1970), and for electrons with sufficiently low energies the losses can be approximated as

$$
\frac{d E}{d t}=-c \sigma_{T}\left(w_{\mathrm{ph}}+\frac{H^{2}}{8 \pi}\right)\left(\frac{E}{m c^{2}}\right)^{2}=-\beta(H) E^{2},
$$

where $\sigma_{T}$ is the Thomson cross-section, $w_{\mathrm{ph}}$ is the total density of the background photons in the central part of the Galactic halo which is taken to be about $2 \mathrm{eV} \mathrm{cm}^{-3}$ (see, e.g., Ackermann et al. 2011, 2012), and $H$ is the magnetic field strength whose value will be derived below.

The energy of the secondary electrons, $E_{e}$, is proportional to the energy of the primary protons, $E_{p}, E_{e} \simeq 0.039 E_{p}$. Then the production rate of secondary electrons for protons of energies above the reaction threshold can be presented as (for details, see Atoyan 1992)

$$
Q\left(E_{e}\right) \simeq \frac{n_{H} c \sigma_{p p}}{0.039} N_{p}\left(E_{e} / 0.039\right)
$$

From Equation (6) we obtain

$$
N_{s e}\left(E_{e}\right) \simeq E_{e}^{-3} \frac{K_{p} n_{H} c \sigma_{p p}}{0.039 \beta(H)}=K_{s e} E_{e}^{-3} .
$$

These electrons also contribute to the total flux of gamma-rays from the FBs by inverse-Compton scattering. The gamma-ray intensity in the direction of the Galactic coordinates $(\ell, b)$ contributed by the $\mathrm{FB}$ is

$$
\begin{aligned}
I_{\gamma}\left(E_{\gamma}, \ell, b\right)= & \frac{c}{4 \pi} \int_{L(\ell, b)} d L \int_{\epsilon} n(\epsilon, r) d \epsilon \\
& \times \int_{p} p^{2} f(r, p, t)\left(\frac{d^{2} \sigma}{d \epsilon d p}\right)_{K N} d p,
\end{aligned}
$$

where $L(\ell, b)$ is the line of sight in the direction $(\ell, b), \sigma_{K N}$ is the Klein-Nishina cross-section (see Blumenthal \& Gould $1970), \epsilon$ is the energy of background photons, and $n(\epsilon)$ is their density.

Below we define the parameters through which the hadronic model can be reanalyzed.

\section{ORIGIN OF THE RADIO AND GAMMA-RAY EMISSION FROM THE FB IN THE HADRONIC MODEL WITH AN ARBITRARY FLUX OF PRIMARY ELECTRONS}

Because the characteristic time of electron energy losses is much shorter than the lifetime of CRs in the bubble $\left(10^{10} \mathrm{yr}\right)$, from Equations (6)-(9) it follows that the spectrum of secondary electrons in the FBs is

$$
N_{s e} \propto E_{e}^{-3}
$$

which is softer than required by the radio data; see Equation (3). The pure hadronic model is unable to reproduce the FB radio spectrum if the lifetime of secondary electrons is determined by the synchrotron and inverse Compton energy losses. Therefore, an additional component of "primary" electrons with a hard spectrum is necessary in order to reproduce the observed radio emission from the FBs.

Because the spectral index of the microwave spectrum is around 0.5 (see Equation (2)), the spectrum of primary electrons 
cannot be steeper than $E_{e}^{-2}$. Therefore, we take it in the arbitrary form

$$
N_{p e}\left(E_{e}\right)=K_{p e} E_{e}^{-2} \theta\left(E_{\max }-E_{e}\right),
$$

where $\theta(x)$ is the Heaviside function.

The intensity of synchrotron emission in the direction $\ell$ is described by the equation (for details, see Ginzburg 1979)

$$
I(v)=\frac{\sqrt{3} e^{3} H L}{m c^{2}} \int_{0}^{E_{\max }} N\left(E_{e},\right) d E_{e} \frac{v}{v_{c}} \int_{\nu / v_{c}}^{\infty} K_{5 / 3}(x) d x,
$$

where $N\left(E_{e}\right)$ is the density of electrons with the energy $E_{e}$, $K_{\alpha}(x)$ is the McDonald function, and $v_{c}=3 e H \gamma^{2} / 4 m c$, where $\gamma$ is the Lorenz factor of an electron.

Below, for estimates, we use approximations for the synchrotron emission of primary and secondary electrons in the form (for details, see Ginzburg 1979)

$$
\begin{aligned}
& I_{p}(v)=K_{p e} 0.103 \frac{e^{3}}{m c^{2}}\left(\frac{3 e}{4 \pi m^{3} c^{5}}\right)^{0.5} H^{3 / 2} L v^{-0.5}=K_{p e} I_{p}^{0}(v), \\
& I_{s}(v)=K_{s e} 0.074 \frac{e^{3}}{m c^{2}}\left(\frac{3 e}{4 \pi m^{3} c^{5}}\right) H^{2} L v^{-1}=K_{s e} I_{s}^{0}(v) .
\end{aligned}
$$

For estimates we take the microwave spectrum from Ade et al. (2013), which gives the intensity $I$

$$
4.3 \cdot 10^{-19} \leqslant I_{23} \leqslant 4.6 \cdot 10^{-19} \mathrm{erg} \mathrm{cm}^{-2} \mathrm{~s}^{-1} \mathrm{sr}^{-1} \text { at } 23 \mathrm{GHz} \text {, }
$$

$2.4 \cdot 10^{-19} \leqslant I_{61} \leqslant 2.7 \cdot 10^{-19} \mathrm{erg} \mathrm{cm}^{-2} \mathrm{~s}^{-1} \mathrm{sr}^{-1}$ at $61 \mathrm{GHz}$.

Then we have

$$
\begin{aligned}
& I_{23}=K_{s e} I_{s}^{0}(23 \mathrm{GHz})+K_{p e} I_{p}^{0}(23 \mathrm{GHz}), \\
& I_{61}=K_{s e} I_{s}^{0}(61 \mathrm{GHz})+K_{p e} I_{p}^{0}(61 \mathrm{GHz}),
\end{aligned}
$$

which give

$$
\begin{aligned}
& K_{s e}(H)=\frac{I_{23} I_{p}^{0}(61)-I_{61} I_{p}^{0}(23)}{I_{s}^{0}(23) I_{p}^{0}(61)-I_{s}^{0}(61) I_{p}^{0}(23)} \\
& K_{p e}(H)=\frac{I_{23} I_{s}^{0}(61)-I_{61} I_{s}^{0}(23)}{I_{p}^{0}(23) I_{e}^{0}(61)-I_{p}^{0}(61) I_{e}^{0}(23)} .
\end{aligned}
$$

The magnetic field strength $H$ can be estimated from the measured intensity of gamma-rays from the FBs (see Su et al. 2010) which is $E_{\gamma}^{2} I_{\gamma}\left(E_{\gamma}\right) \simeq 4.2 \times 10^{-7} \mathrm{GeV} \mathrm{cm}^{-2} \mathrm{~s}^{-1} \mathrm{sr}^{-1}$. From the approximation formulae for proton gamma-ray production by $p p$ collisions (see Atoyan 1992) and by inverse-Compton scattering of primary electrons off background photons (see Ginzburg 1979) the equation for the total gamma-ray emission is (here we neglected the contribution from secondary electrons)

$$
\begin{aligned}
E_{\gamma}^{2} I_{\gamma}\left(E_{\gamma}\right) \simeq & \frac{c L}{4 \pi}\left(K_{p}(H) 0.075 n_{H} \sigma_{p p}+K_{p e}(H) \sigma_{T} n_{p h}\right. \\
& \left.\times \frac{\left(4 / 3 \varepsilon_{p h} E_{\gamma}\right)^{0.5}}{m c^{2}}\right)
\end{aligned}
$$

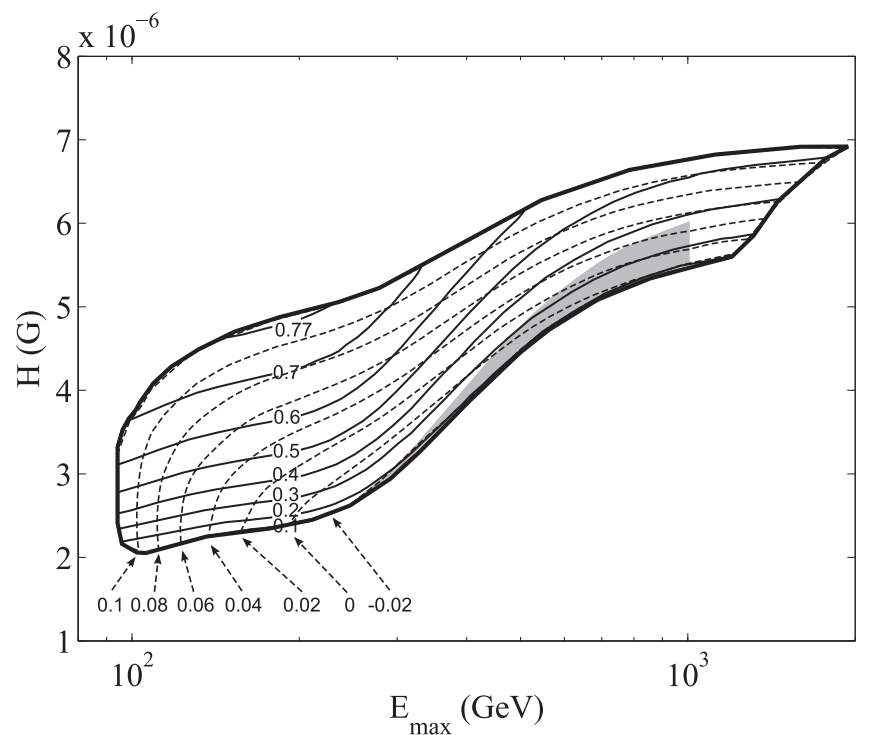

Figure 1. Contours of $\chi_{p p}=$ const (solid lines) and contours of deviations of the model spectral index $\alpha$ from $\bar{\alpha}=0.56, \Delta \alpha=\alpha-\bar{\alpha}=$ const (dashed lines). The spectral index $\bar{\alpha}=0.56$ is derived by Ade et al. (2013) from the Planck data. The shaded area shows the region of the parameters for the pure leptonic model.

As an example we take the intensity of gamma-ray emission at $E_{\gamma}=1 \mathrm{GeV}$ which is produced by inverse-Compton scattering off the relic photons $\left(\varepsilon_{\mathrm{ph}} \simeq 6.6 \times 10^{-4} \mathrm{eV}, n_{\mathrm{ph}} \simeq 400 \mathrm{~cm}^{-3}\right)$. For the most favorable parameters for the proton contribution (the steepest spectrum of the gamma-rays within error bars), we obtain from Equation (23) that the required magnetic field in the FBs in this case is $H \sim 1.2 \times 10^{-5}$. The upper limit of the contribution of protons to the total flux is about $36 \%$ while the rest, $64 \%$, are produced by inverse-Compton scattering of primary electrons off the relic photons. These estimates were derived from Equation (23) in which we used analytical approximations of the gamma-rays produced by electrons and protons with power-law spectra. Further, the cross-sections of these processes were taken as constants. More accurate results for arbitrary spectra of emitting particles can be obtained from numerical calculations with the cross-sections from Blumenthal \& Gould (1970) and Kamae et al. (2006), which in this case are functions of particle energy. Below we define the fraction of the FB gamma-rays produced by protons as $\chi_{p p}$. From the inequalities (17) and (18), we calculate numerically the maximum value of $\bar{\chi}_{p p}$ for a given magnetic field strength $H$ and the cutoff energy of primary electrons $E_{\max }$ although other values of $\chi_{p p}<\bar{\chi}_{p p}$ are possible within the limits of the inequalities. The result of the numerical calculation of the contribution from primary protons is shown in Figure 1. The solid lines show the maximum fraction of the FB gamma-rays produced by protons, $\bar{\chi}_{p p}$, as a function of the magnetic field strength $H$ and the cutoff energy of primary electrons $E_{\max }$.

We also showed in this figure variations of $\Delta \alpha=\alpha-\bar{\alpha}$, where $\alpha$ is the model spectral index of microwave emission derived for different $H$ and $E_{\max }$ and $\bar{\alpha}=0.56$ is obtained by Ade et al. (2013) from the Planck data. The range of the model index $\alpha$ in the frequency interval from 23 to $61 \mathrm{GHz}$ is restricted between 0.48 and 0.67 , following from the inequalities (17) and (18). From the figure we see that the higher the value of $\alpha$, the larger $\chi_{p p}($ see Figure 1$)$.

The maximum proton contribution (for $\alpha=0.67$ ) is about $78 \%$ if the magnetic field strength is $H \simeq 5 \times 10^{-6} \mathrm{G}$, which 

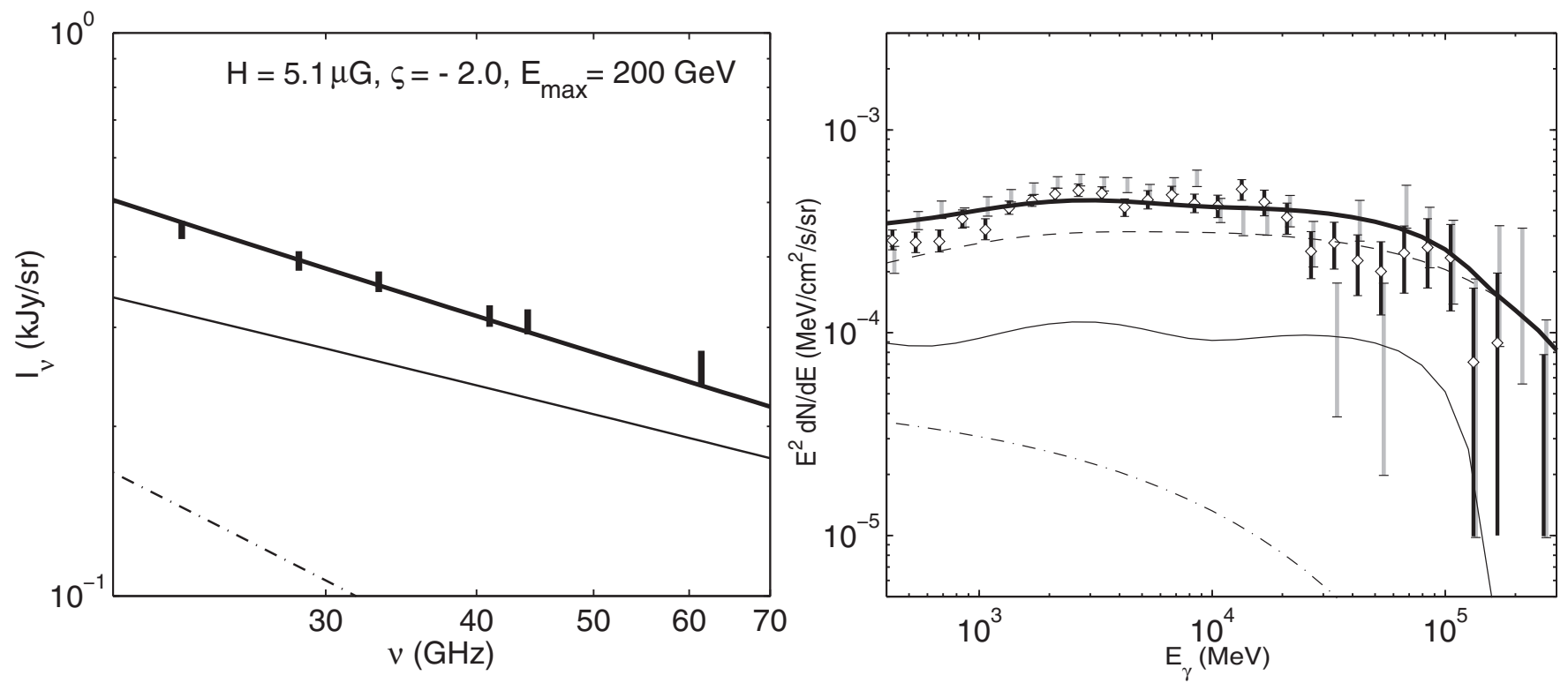

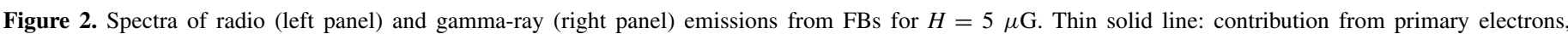

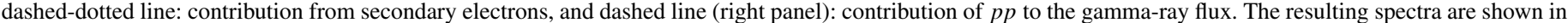

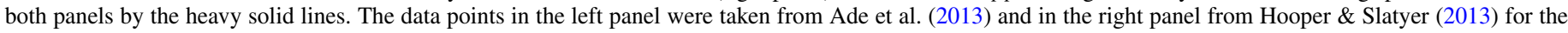
latitude range $20^{\circ}-30^{\circ}$ (gray lines) and for the range $30^{\circ}-40^{\circ}$ (black lines with diamonds).

is about two times larger than what follows from Equation (23). The reasons for this discrepancy are simplifications of analytical equations describing gamma-ray production as we mentioned above.

The thick solid line shows the area of permitted parameters when the hadronic model reproduces the FB microwave and gamma-ray fluxes. For each value of $E_{\max }$ the range of permitted values of $H$ is restricted. Outside this area the model is unable to satisfy Equations (17) and (18) simultaneously. The shaded area shows the range of parameters when the origin of these fluxes is purely leptonic when the microwave spectrum is close to $v^{-0.5}$. The pure leptonic model corresponds to the condition $\chi_{p p}=0$. Within limits of the inequalities (17) and (18) the condition $\chi_{p p}=0$ is satisfied for different $H$ and $E_{\max }$, the values of which are shown by the shaded area.

We notice that in our analysis that we accepted higher deviations from $\bar{\alpha}$ than derived by Ade et al. (2013), $\bar{\alpha}=$ $0.56 \pm 0.05$, increasing the permitted value of $\chi_{p p}$.

The magnetic field strength in the FBs is quite uncertain. Thoudam (2013) estimated its value from the GALPROP program, which gave $1.3 \mu \mathrm{G}$ at an altitude of $5 \mathrm{kpc}$ above the Galactic plane. The estimates of the magnetic field inside the Bubbles by Jones et al. (2012) and Carretti et al. (2013) ranges from 6 to $15 \mu \mathrm{G}$.

The spectra of radio and gamma-ray emission for $H \sim$ $5 \mu \mathrm{G}$ are shown in Figure 2. We see that even in the most favorable case for the hadronic model (when the magnetic field strength is about $5 \mu \mathrm{G}$ ) the contribution from protons ( $p p$ collisions + IC from secondary electrons/positrons) is about $80 \%$, and the remaining $20 \%$ is produced by primary electrons. The gamma-ray flux from $p p$ collisions is $3.2 \times$ $10^{-9} \mathrm{erg} \mathrm{s}^{-1} \mathrm{~cm}^{-2} \mathrm{sr}^{-1}$, the flux from secondary electrons is $2.5 \times 10^{-10} \mathrm{erg} \mathrm{s}^{-1} \mathrm{~cm}^{-2} \mathrm{sr}^{-1}$, and the necessary flux from primary electrons is $1.1 \times 10^{-9} \mathrm{erg} \mathrm{s}^{-1} \mathrm{~cm}^{-2} \mathrm{sr}^{-1}$. Thus, we conclude that a purely hadronic origin of the nonthermal emission (gamma and radio) from the FBs is problematic.

\section{EFFECT OF ADIABATIC ENERGY LOSSES}

As we see from the previous section the pure hadronic model is unable to reproduce both radio and gamma-ray emission from the FBs if secondary electrons lose their energy by synchrotron and inverse-Compton emission. The radio spectrum of secondary electrons is too soft, and an additional arbitrary component of electrons has a relatively hard spectrum.

However, the situation is different if adiabatic losses are significant in the FBs. For a divergent outflow from the GC region with the velocity $\mathbf{u}$, the rate of adiabatic energy losses is $d E / d t=-E \nabla \cdot \mathbf{u} / 3$.

There are arguments, indeed, in favor of plasma outflow from the Galactic central region (see Crocker \& Aharonian 2011; Carretti et al. 2013). Numerical and analytical calculations of Breitschwerdt et al. $(1991,2002)$ showed that the outflow velocity in the halo increased linearly with the altitude $z$ above the Galactic plane. Thus, we can approximate the velocity distribution in the form $u(z)=3 \lambda z$, and the rate of adiabatic and synchro-Compton losses has the form $d E / d t=-\left(\lambda E+\beta E^{2}\right)$. From Equation (8), we obtain that

$$
N_{s e}=\frac{Q}{\lambda(\varsigma+1)} \frac{E^{-\varsigma}}{(1+\beta E / \lambda)},
$$

where the source function was taken to be in the form of Equation (4). We see that for energies $E<\lambda / \beta$ the spectrum of secondary electrons is flat, which is necessary for the observed radio emission from the FBs and an additional component of primary electrons is unnecessary in this case.

If the velocity gradient is relatively small, then a component of primary electrons is still required in order to reproduce the radio data. However, our numerical calculations show that the fraction of radio emission from secondary electrons increases with the increase of the outflow gradient velocity $\lambda$ as shown in Figure 3 (left panel). The value of $\chi_{R}$ in this figure shows the fraction of the radio flux from the FBs produced by the secondary electrons. 

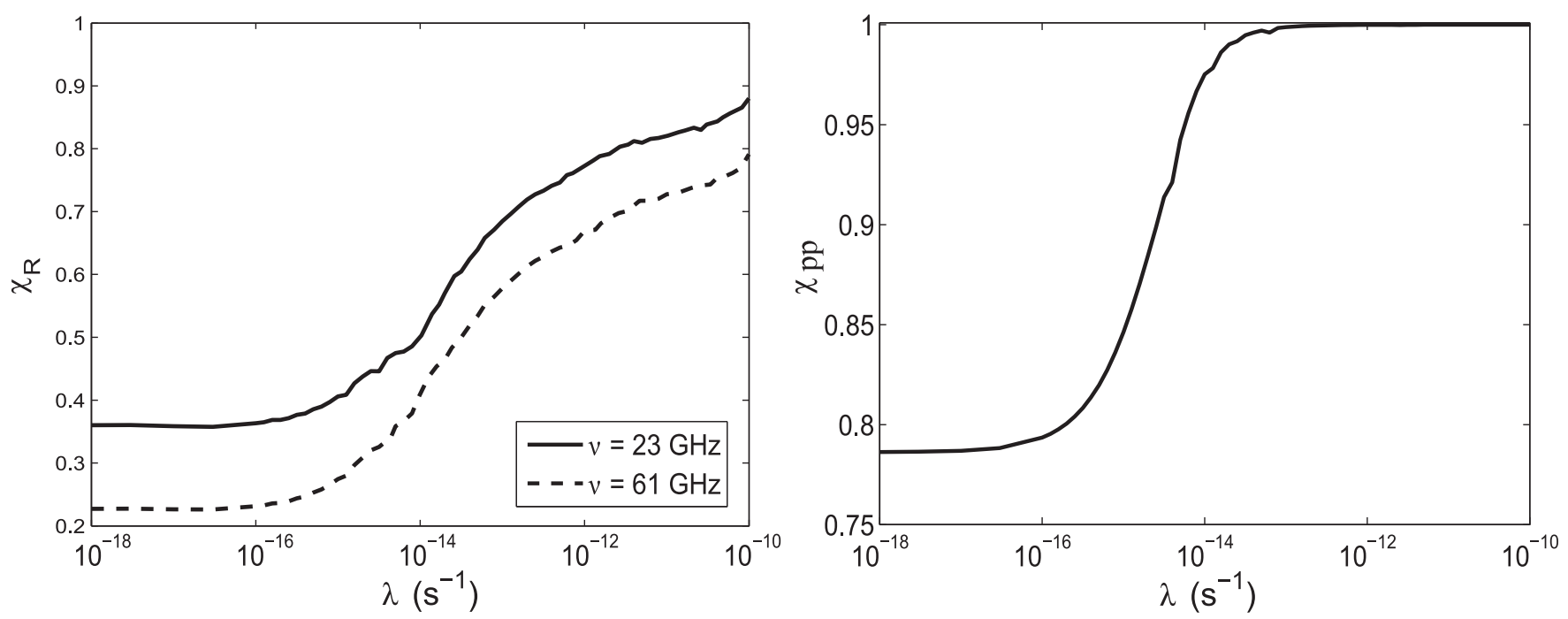

Figure 3. Left panel: the fraction of radio flux from the FBs at frequencies 23 and $61 \mathrm{GHz}$ which is produced by the secondary electrons as a function of the velocity gradient $\lambda$. The total radio flux from the FBs was taken from Ade et al. (2013). Right panel: the fraction of gamma-ray flux from the FBs produced by $p p$ collisions as a function of the velocity gradient $\lambda$.
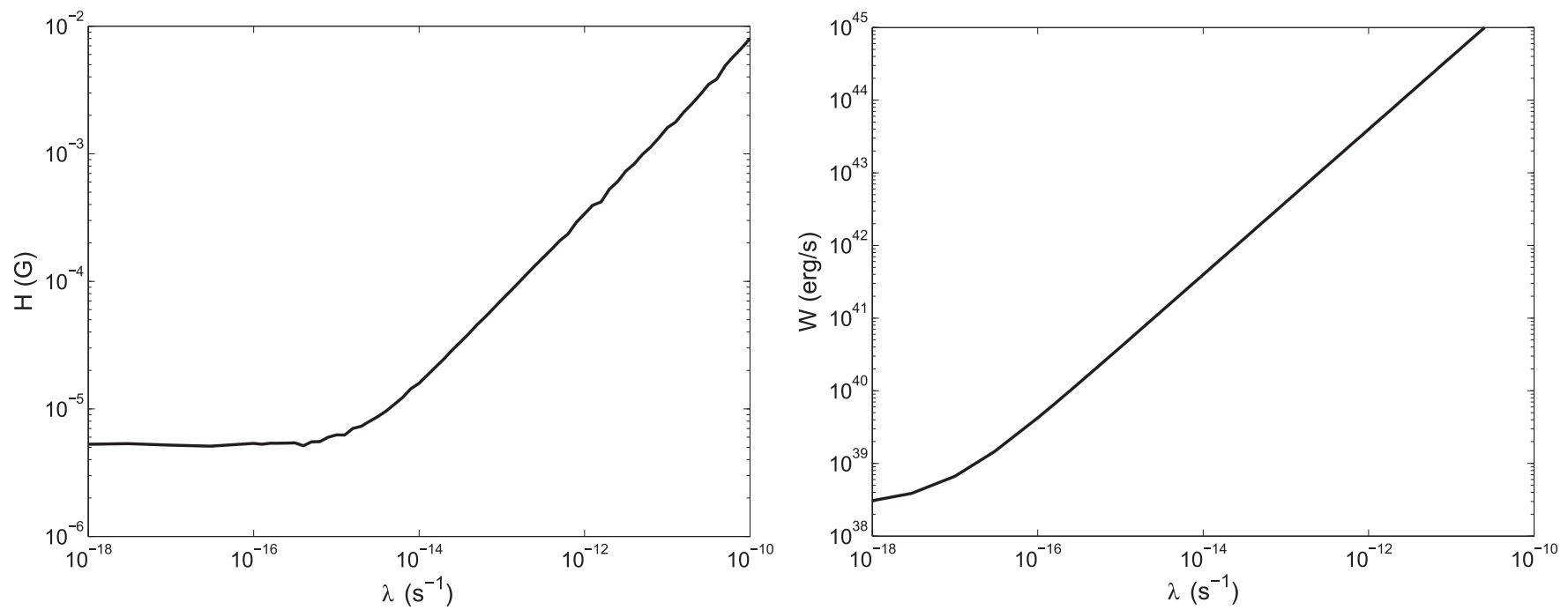

Figure 4. Left panel: the strength of the magnetic field required for the observed radio flux from the FBs as a function of the velocity gradient $\lambda$. Right panel: the power of CR sources required to produce the gamma-ray flux from the FBs by $p p$ collisions as a function of the velocity gradient $\lambda$.

The other part $\left(1-\chi_{R}\right)$ of the flux is generated by primary electrons.

The fraction of the total gamma-ray flux produced by $p p$ collisions in the model with the outflow is shown in Figure 3 (right panel). As we can see the hadronic model with adiabatic losses describes quite well the gamma and radio emission from the FB for very large $\lambda$, and in the limit $\lambda \gg 10^{-10} \mathrm{~s}^{-1}$ no additional component of primary electrons is needed.

However, the density of CRs drops for high velocity gradients $\lambda$ (see Equation (24)). Therefore, for high $\lambda$, a stronger magnetic field is necessary in order to produce the observed radio flux from the FBs and the higher power of CR sources for the $p p$ gamma-ray flux there. This effect is illustrated in Figure 4 (left and right panels).

From these figures we see that the hadronic model with an outflow requires an unrealistically high magnetic field strength and CR power in the FBs in order to reproduce the observed gamma and radio fluxes there. In our opinion this is a serious problem of the model.

\section{CONCLUSION}

The conclusions can be itemized as follows.

1. The pure hadronic model is unable to reproduce the gammaray and radio fluxes from the FBs because the secondary electrons have too-soft spectrum if they lose energy by synchrotron radiation and inverse-Compton scattering. In order to obtain the observed radio emission in this model an additional component of primary electrons with a hard spectrum is necessary, or very effective adiabatic losses are required.

2. The additional component of primary electrons contributes to the total gamma-ray flux from the FBs by inverseCompton scattering. The relation between components produced by protons and primary electrons depend on the magnetic field strength in the FB and the spectral index of primary electrons.

3. For the spectrum of primary electrons $\propto E^{-2}$, the $p p$ collisions can only provide about $80 \%$ of the FB 
gamma-ray flux under the most favorable conditions when $H \simeq 5 \mu \mathrm{G}$.

4. If the magnetic field strength is larger than $7 \mu \mathrm{G}$ then neither the hadronic nor the leptonic models are able to reproduce gamma-ray and radio emission from the FBs if the spectrum of primary electrons is $\propto E^{-2}$. For a harder spectrum, e.g., as $E^{-1}$, a mixture of the hadronic and leptonic model is able to reproduce the observed gamma and radio emission from the FBs even for $H>10 \mu \mathrm{G}$.

5. With a decrease of the magnetic field strength, the contribution from primary electrons to the total FB gamma-ray flux increases, and at $H \simeq 2.5 \mu \mathrm{G}$ the origin of gamma-rays from the FB is purely leptonic.

6. In principle, the pure hadronic model is able to reproduce the spectra of radio and gamma-ray emission from the FBs under the conditions of a strong plasma outflow from the $\mathrm{GC}$ when the rate of adiabatic loss exceeds losses from synchrotron radiation and inverse-Compton scattering. The spectrum of secondary electrons in this case is relatively hard, $\propto E^{-2}$, which is necessary for the observed radio emission from the FBs.

7. However, in this case the required values of the magnetic field strength in the FBs and the power of CR sources are much higher than those following from observations. We conclude that all versions of the hadronic model of the FBs, which we analyzed, are in our opinion problematic. In any case it is not easy to reproduce characteristics of radio and gamma-ray emission in the framework of this model.

K.S.C. is supported by the GRF grants of the Government of the Hong Kong SAR under HKU 701013. D.O.C. is supported in part by the LPI Educational-Scientific Complex and Dynasty Foundation. D.O.C. and V.A.D. acknowledge support from RFFI grants 12-02-00005, 15-52-52004, 15-02-02358, and 15-02-08143. C.M.K. is supported, in part, by the Taiwan
Ministry of Science and Technology grant MOST 102-2112M-008-019-MY3. K.S.C., D.O.C., and V.A.D. acknowledge support from the International Space Science Institute to the International Team "New Approach to Active Processes in Central Regions of Galaxies.”

\section{REFERENCES}

Ackermann, M., Ajello, M., Allafort, A., et al. 2011, Sci, 334, 1103 Ackermann, M., Ajello, M., Atwood, W. B., et al. 2012, ApJ, 750, 3 Ackermann, M., Albert, A., Atwood, W. B., et al. 2014, ApJ, 793, 64

Ade, P. A. R., Aghanim, N., Arnaud, M., et al. (Planck Collaboration) 2013, A\&A, 554, 139

Atoyan, A. M. 1992, A\&A, 257, 476

Berezinskii, V. S., Bulanov, S. V., Dogiel, V. A., Ginzburg, V. L., \& Ptuskin, V. S 1990, in Astrophysics of Cosmic Rays ed. V. L. Ginzburg (Norht-Holland: Amsterdam)

Blumenthal, G. R., \& Gould, R. J. 1970, RvMPh, 42, 237

Breitschwerdt, D., Dogiel, V. A., \& Völk, H. J. 2002, A\&A, 385, 216

Breitschwerdt, D., McKenzie, J. F., \& Völk, H. J. 1991, A\&A, 245, 79

Carretti, E., Crocker, R. M., Staveley-Smith, L., et al. 2013, Natur, 493, 66

Cheng, K.-S., Chernyshov, D. O., Dogiel, V. A., \& Ko, C.-M. 2014, ApJ, 790, 23

Crocker, R. M., \& Aharonian, F. 2011, PRL, 106, 101102

Crocker, R. M., Bicknell, G. V., Carretti, E., et al. 2013, ApJL, 791, L20

Crocker, R. M., Jones, D. I., Aharonian, F., et al. 2011, MNRAS, 413, 763

Dobler, G., Finkbeiner, D. P., Cholis, I., et al. 2010, ApJ, 717, 825

Finkbeiner, D. P. 2004, ApJ, 614, 186

Fujita, Y., Ohira, Y., \& Yamazaki, R. 2013, ApJL, 775, L20

Ginzburg, V. L. 1979, Theoretical Physics and Astrophysics (International Series in Natural Philosophy, Vol. 99; Oxford: Pergamon)

Hooper, D., \& Slatyer, T. R. 2013, Phys. Dark Univ., 2, 118

Jones, D. I., Crocker, R. M., Reich, W., Ott, J., \& Aharonian, F. A. 2012, ApJL, 747, L12

Kamae, T., Karlsson, N., Mizuno, T., et al. 2006, ApJ, 647, 692

Mertsch, P., \& Sarkar, S. 2011, PhRvL, 107, 091101

Shibata, T., Ohira, Y., Kohri, K., \& Yamazaki, R. 2014, APh, 55, 8

Su, M., Slatyer, T. R., \& Finkbeiner, D. P. 2010, ApJ, 724, 1044

Thoudam, S. 2013, ApJL, 778, L20

Yang, H.-Y. K., Ruszkowski, M., Ricker, P. M., Zweibel, E., \& Lee, D. 2012, ApJ, 761,185

Yang, R.-Z., Aharonian, F., \& Crocker, R. 2014, A\&A, 567, A19 